\title{
Family function and eating behaviours among Hispanic/Latino youth: results from the Hispanic Community Children's Health Study/Study of Latino Youth (SOL Youth)
}

\author{
U Colón-Ramos ${ }^{1, *}$, R Monge-Rojas ${ }^{2}$, V Smith-Castro ${ }^{3}$, J Wang ${ }^{4}, \mathrm{YI} \mathrm{Cheng}^{4}, \mathrm{KM}$ Perreira $^{5}$, \\ L Van Horn ${ }^{6}$, D Sotres-Alvarez ${ }^{5}$, CR Isasi ${ }^{7}$ and LC Gallo ${ }^{8}$ \\ 'Department of Global Health, Milken Institute School of Public Health at the George Washington University, \\ Washington, DC 20037, USA: ${ }^{2}$ Instituto Costarricense de Investigación y Enseñanza en Nutrición y Salud \\ (INCIENSA), Tres Ríos, Cartago, Costa Rica: ${ }^{3}$ Universidad de Costa Rica, Ciudad de la Investigación, Facultad de \\ Ciencias Sociales, San Pedro, Costa Rica: ${ }^{4}$ Center for Translational Science, Children's Research Institute at Children's \\ National Health System, Silver Spring, MD, USA: ${ }^{5}$ Department of Biostatistics, Collaborative Studies Coordinating \\ Center, University of North Carolina at Chapel Hill, Chapel Hill, NC, USA: ${ }^{\circ}$ Department of Preventive Medicine, \\ Feinberg School of Medicine, Northwestern University, Chicago, IL, USA: ${ }^{7}$ Department of Epidemiology and \\ Population Health, Albert Einstein College of Medicine, Bronx, NY, USA: ${ }^{8}$ Department of Psychology, San Diego State \\ University, South Bay Latino Research Center, Chula Vista, CA, USA
}

Submitted 23 August 2019: Final revision received 20 March 2020: Accepted 16 April 2020: First published online 25 August 2020

\begin{abstract}
Objective: To elucidate mechanisms across family function, home environment and eating behaviours within sociocultural context among Hispanic youth.

Design: Two models tested via path analysis (youth fruit and vegetable (FV) consumption; empty energy consumption) using data from the Study of Latino Youth (2011-2013).

Setting: Chicago, IL; Miami, FL; Bronx, NY; San Diego, CA.

Participants: Youth (8-16-year-olds), n 1466.

Results: Youth ate 2.4 servings of FV per d and received $27 \%$ of total energy from empty energies. Perceiving higher acculturative stress was indirectly associated with lower FV consumption via a pathway of low family function and family support for FV $(\beta=-0.013, P<0 \cdot 001)$ and via lower family closeness and family support $(\beta=-0.004, P=0.004)$. Being $>12$-year-olds was indirectly associated with lower FV consumption via lower family closeness and family support $(\beta=-0.006, P<0.001)$. Household food security was indirectly associated with greater FV consumption via family closeness and family support $(\beta=0.005$, $P=0.003$ ). In contrast, perceiving higher acculturative stress was indirectly associated with higher empty energy consumption (via family closeness and family support: $\beta=0.003, P=0.028$ and via low family function and low family support: $\beta=0.008, P=0.05)$. Being older was associated with higher consumption of empty energies via family closeness (related to family support: $\beta=0 \cdot 04, P=0 \cdot 016$; parenting strategies for eating: $\beta=0.002, P=0.049$ ).

Conclusions: Findings suggest pathways of influence across demographic and sociocultural context, family dynamics and home environment. The directionality of these associations needs confirmation using longitudinal data.
\end{abstract}

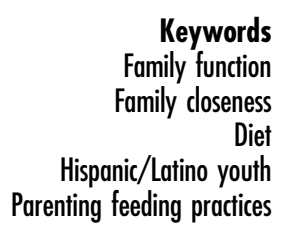

Keywords Family function Family closeness Hispanic/Latino youth Parenting feeding practices
Prevalence of obesity and diabetes among youth has been escalating globally, and Hispanics/Latinos (hereafter 'Hispanics') are disproportionately burdened by these diseases. Among youth 2-19-year-olds, obesity prevalence is higher among Hispanics at 21.9 v. $14.7 \%$ for non-Hispanic whites in the USA ${ }^{(1)}$. By the time they reach adolescence,
Hispanics have higher rates of insulin resistance and impaired fasting glucose levels than non-Hispanic white youth ${ }^{(2)}$.

The importance of adopting a healthier diet to prevent obesity and diet-related chronic disease is well established. Consuming fruits and vegetables (FV) contributes to the intake of dietary fibre and of foods with lower glycaemic 
index, which help prevent obesity and diet-related chronic diseases $^{(3-6)}$. The 2015 Dietary Guidelines for Americans recommend that people reduce their consumption of 'empty energies' (energies from solid fats, added sugars and alcohol) because they do not provide essential nutrients and can displace more nutrient-dense foods ${ }^{(7,8)}$. However, there is limited information on how to best support these dietary behaviours among youth.

The literature consistently documents that eating behaviours among youth are shaped by the environment at home, especially parental food practices and parenting styles $^{(9-13)}$. These practices influence children's attitudes and beliefs about foods ${ }^{(13)}$. For example, parenting practices related to pressuring children to eat are consistently associated with greater obesogenic dietary intake ${ }^{(14)}$. Parenting styles that describe parent behaviours around child-rearing goals are also associated with obesogenic diets $^{(15)}$. Authoritative parenting styles (high responsiveness to, and high demandingness of, youth) are associated with healthier weight and better diet among youth, whereas authoritarian and permissive parenting styles have been associated with unhealthy eating ${ }^{(16-23)}$. Nonetheless, the majority of these studies were conducted on nonHispanic white populations, with more limited and equivocal findings about practices ${ }^{(24,25)}$ and parenting styles ${ }^{(26-33)}$ among Hispanics. Some investigators posit that Hispanic parenting styles and practices are sometimes nonconforming with parenting styles among the dominant US culture, emphasising the need for further research on the familial, sociocultural, psychological and economic context in which parenting practices and styles occur ${ }^{(34,35)}$.

According to Family Systems Theory, food parenting practices and styles are impacted by family dynamics and interactions ${ }^{(36)}$. Children's positive feelings about how the family behaves (family function ${ }^{(37,38)}$ ) and their perceptions about the warmth and love that they receive from their parents (family closeness ${ }^{(39)}$ ) are associated with better diet in some ${ }^{(40,41)}$, but not all ${ }^{(42)}$, studies. How family dynamics may influence parenting practices and styles, especially those related to feeding, as well as their offspring's eating behaviours are not well understood. A family that functions weakly, with conflict, less structure or less warmth may wish to spend less time together, is more likely to purchase foods away from the home and/or may be less aware of the dietary needs of the family members and therefore has less healthful items available at home.

Family dynamics may be influenced by the sociocultural, psychological and economic contexts of the household. For example, integration (bicultural orientation) has been associated with higher diet quality among Hispanic youth compared with youth who were assimilated to the $\mathrm{USA}^{(43)}$. Ethnicity, sociocultural norms and food insecurity may also influence parents' attitudes and practices towards child rearing and have been associated with child health or eating behaviours ${ }^{(44-46)}$. The role that these contextual characteristics may play in family dynamics and youth diet warrants further investigation in order to guide interventions that aim to promote healthful eating among youth. With the renewed emphasis on the interactions between multiple complex factors that influence dietary choices beyond personal choice ${ }^{(47)}$, there is an increased interest in elucidating the mechanisms between sociocultural context, family and home environments that may influence diet among Hispanic youth.

Drawing from Family Systems Theory ${ }^{(36)}$ and the socialecological approach ${ }^{(48)}$, the current study tested a model that posits that the socio-demographic and sociocultural context in which families live influence the home environment via family dynamics, and subsequently youth eating behaviours (Fig. 1).

\section{Methods}

The current study used cross-sectional data from Hispanic Community Children's Health Study/Study of Latino Youth

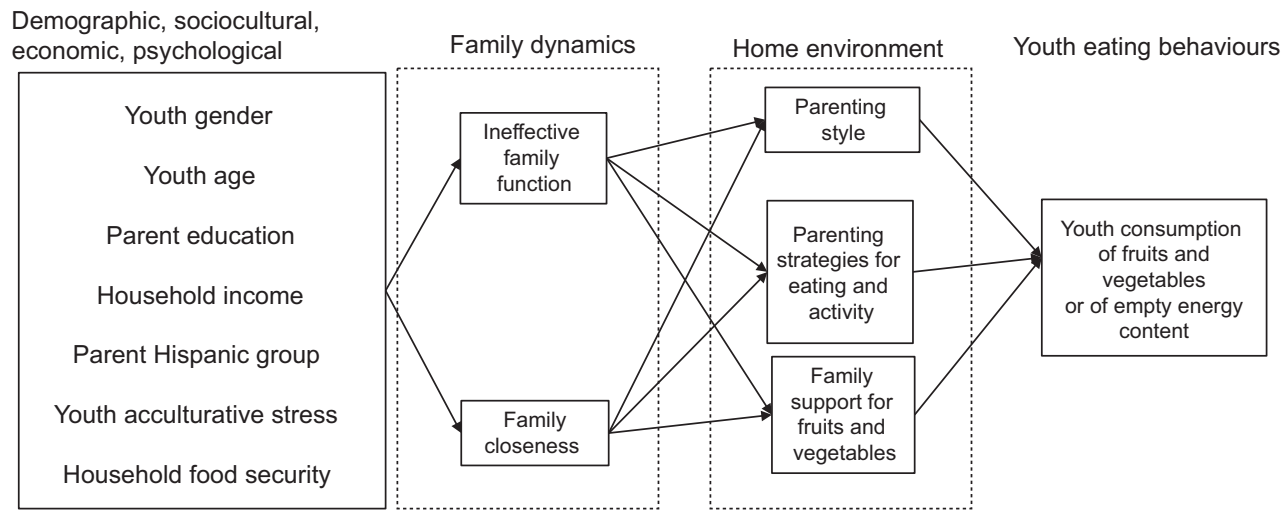

Fig. 1 Conceptual model depicting the proposed effects of demographic, sociocultural, economic and psychological variables on youth eating behaviours indirectly through family dynamics and home environment among Hispanic/Latino youth. Study of Latino Youth 
(SOL Youth) to test the conceptual model depicted in Fig. 1 via path analysis. Figure 1 posits that demographic, sociocultural, psychological and economic contexts of families influence family dynamics, which in turn relate to youth eating behaviours (consumption of FV as well as empty energies) via the home environment. Although the constructs of family dynamics are part of the many characteristics of the home environment, in this model, we intentionally separate family dynamics from home feeding environment to test if closeness/function affects parenting feeding practices and styles at home. Taking into account that these data are cross-sectional, we chose to use the terminology 'mediation path', 'influence' or 'effect' in these path analyses. A line with a single arrow represents a direct effect (relationship) between two variables with the head of the arrow pointing towards the variable being influenced by another variable.

\section{Data}

Data were from 1466 Hispanic youths (aged 8-16 years) enrolled in SOL Youth between 2011 and $2013^{(49)}$. SOL Youth is an ancillary study of youth enrolled in the parent population-based study Hispanic Community Health Study (HCHS)/SOL ${ }^{(50)}$. HCHS/SOL participants were recruited from four US cities (Chicago, IL; Miami, FL; Bronx, NY and San Diego, CA) using probability sampling. Details about the methodology and protocols of SOL Youth have been described and published elsewhere ${ }^{(49-52)}$. SOL Youth included comprehensive measures for youth eating behaviours, food-related home environment, family dynamics and sociocultural and psychosocial characteristics among varied Hispanic background groups. Written informed consent and assent were obtained from parent/caregivers and their offspring, respectively. The study was conducted with approval from the institutional review boards of each of the institutions involved in the study.

\section{Outcomes}

Two dietary outcomes were examined in the current study: youth FV intake (servings/d) and percentage of empty energies (percentage from energy) consumed. Both outcomes were assessed by two interview-administered 24-h dietary recalls using the multi-pass method with the Nutrition Data System for Research software developed by the University of Minnesota(53). Nutrition Data System for Research contains over 18000 foods and 8000 brand-name products and many Hispanic and Latino foods. Additional Hispanic/Latino foods were also added as new foods were reported by the diverse HCHS/SOL population. Total intake was calculated as the average of two 24-h dietary recalls. Fruits (without fruit juices) included whole fruits, fried fruits and fruit-based savoury snacks, as per established criteria ${ }^{(54)}$. Vegetables (excluding fried potatoes) included dark-green, deep-yellow, tomatoes, white potatoes, other starchy vegetables, legumes, vegetable juice and other vegetables as per established criteria. FV were measured in standard serving sizes as detailed elsewhere ${ }^{(54)}$. Following the Healthy Eating Index 2010, empty energies (expressed as percentage of total energies) were defined as energies from solid fats, added sugars and alcohol beyond moderate amounts) ${ }^{(55,56)}$. Alcohol was kept in the definition of empty energies because it could be a potential contributor, as $19 \%$ of SOL youth reported that they had ever used alcohol and $6 \%$ had used alcohol in the past $30 \mathrm{~d}^{(57)}$.

\section{Direct association: bome environment}

The food-related home environment included the constructs of parenting feeding practices and parenting styles $^{(9-12,58)}$. First, Family Support for FV Intake (reported by youth) was measured with four items ${ }^{(59)}$ (Cronbach's $\alpha$ in SOL Youth $=0.76$ ). Second, Parenting Strategies for Eating and Activity Scale (PEAS, reported by youth) was a twenty-six-item scale that asked parents about strategies that they used to encourage healthy youth eating behaviours, exercise and limits around screen time ${ }^{(26)}(\alpha$ in SOL Youth $=0.88$ ). Third, Parenting Styles (reported by parents) were measured with a sixteen-item scale designed to assess responsive and demanding parenting behaviours, with responses ranging from 1 to 4 ( $\alpha$ in SOL Youth $=0 \cdot 84$ ). Per established criteria ${ }^{(60)}$, parents were grouped into four parenting styles based on two subscales of demandingness and responsiveness: 'authoritative' (high in both demandingness and responsiveness), 'neglectful' (low in both demandingness and responsiveness), 'indulgent' (low in demandingness and high in responsiveness) and 'authoritarian' (high in demandingness and low in responsiveness). Consistent with research showing that authoritative parenting relates to healthier outcomes among youth, categories were collapsed to compare authoritative style with all others (e.g. neglectful, indulgent and authoritarian styles).

\section{Indirect influence: family dynamics}

The constructs of family functioning and family closeness were used to capture the bidirectional relationships between family members. Family functioning (reported by youth) was a twelve-item subscale of the McMaster Family Assessment Device ${ }^{(37,38)}$, with responses ranging from strongly agree to strongly disagree in a four-point Likert scale ( $\alpha$ in SOL Youth $=0 \cdot 77$ ). The scale included questions such as 'In the times of crisis we turn to each other for support', 'Individuals are accepted for what they are', 'We confide in each other' and 'We don't get along with each other'. These questions alternated between positive feelings/effective family function and negative feelings/ineffective family function. In the original scale, higher scores indicate ineffective or weaker family functioning. Family closeness (reported by youth) was a sixitem scale that asked them about their closeness to their mother and father, how much they thought these parents care for them and how they were warm and loving towards 
the youth most of the time ${ }^{(39)}(\alpha$ in SOL Youth $=0 \cdot 70)$. This question was asked only for those youth who had a mother and father as the primary caregiver.

\section{Indirect influence: sociocultural, economic and psychological context}

We tested various measures of these contexts, including household food security, acculturative stress and sociodemographic/economic variables. Household food security was measured with six items that asked parents about whether they worried about food running out, not lasting, not affording a balanced meal, relying on low-cost foods or not affording balanced meals for youth ${ }^{(49)}$. Higher scores mean higher food security. Acculturative stress, perceived by youth, was defined as the 'psychological, somatic and social difficulties that accompany the adaptation to the new culture ${ }^{,(61,62)}$. The nine-item acculturative stress index included three components: family acculturation conflicts, discrimination and language conflict ${ }^{(63,64)}$. The higher the score, the higher the acculturative stress. Youth gender and age, and parent-reported: parental education, household annual income and Hispanic group of origin/identity (Mexican, Caribbean (Puerto Rican, Dominican, Cuban) or other (Central/South American/mixed)) were also tested as variables that could influence youth eating behaviours indirectly through their effects on family dynamics. For path analysis models, income was dichotomised into $<\$ 30 \mathrm{k}$ or $>\$ 30 \mathrm{k}$; parental education was dichotomised into high school or less, or more than high school; youth age was dichotomised into $\leq 12$ - or $>12$-year-olds.

\section{Statistical analysis}

To test the model (Fig. 1), we used Mplus $8 \cdot 2^{(65)}$ to estimate a path analysis model, which is an extension of multiple regression. A robust maximum likelihood estimator was used for model estimation. Maximum likelihood estimator provides parameter estimates with standard errors, model $\chi^{2}$ statistic and fit indices that are robust to data non-normality. Maximum likelihood estimator allows an assumption of missing at random, which is more plausible than the assumption of missing completely at random in the traditional analytical methods. In addition, maximum likelihood estimator was implemented in conjunction with full information maximum likelihood for model estimation ${ }^{(65)}$; thus, every piece of information available in the data was used for model estimation ${ }^{(66,67)}$. All paths, including the double mediation paths, were tested using the multivariate delta method ${ }^{(68)}$. Model estimation incorporated stratification, clustering and sampling weights to account for the complex survey design of SOL Youth. Model fit was assessed according to the established criteria ${ }^{(65,69)}$. For each model, only significant direct and indirect effects with estimated standardised coefficients are reported.

\section{Results}

Table 1 presents the descriptive statistics of the sample (unweighted). The majority of the sample was composed of youth >12-year-olds (almost 60\%), who lived with parents of lower education attainment (had high school or less), lower household income $(<\$ 30$ 000) and Mexican descent. On average, youth ate 2.4 servings of $\mathrm{FV}$ per $\mathrm{d}$ and received $27 \%$ of their total energies from empty energies.

\section{Fruits and vegetables model}

Figure 2 depicts the direct (in arrows) and indirect (subscripts) effects of the variables tested in the model. The model fit statistics show that the data fit the model very well $\left(\chi^{2}=0.454 ; \mathrm{df}=2 ; P=0.80 ;\right.$ comparative fit index $(\mathrm{CFI})=1$; Tucker-Lewis index $(\mathrm{TLI})=1$; root mean square error of approximation $($ RMSEA $)=0 \cdot 000,90 \%$ confidence interval

Table 1 Descriptive statistics for all variables used in path analysis. Study of Latino Youth (2011-2013) (unweighted $n$ 1466)

\begin{tabular}{|c|c|c|}
\hline \multicolumn{3}{|l|}{$\begin{array}{l}\text { Sociocultural, economic and psychological } \\
\text { context }\end{array}$} \\
\hline $\begin{array}{l}\text { Household food security }{ }^{\star} \text {, score range 5-15 } \\
\text { (mean and SD) }\end{array}$ & $12 \cdot 60$ & $2 \cdot 70$ \\
\hline $\begin{array}{l}\text { Youth acculturative stress index, score } \\
\text { range } 1-5 \dagger \text { (mean and SD) }\end{array}$ & 1.63 & 0.59 \\
\hline \multicolumn{3}{|l|}{ Youth age $(n$ and \%) } \\
\hline$\leq 12$-year-olds & 844 & 57.57 \\
\hline$>12$-year-olds & 622 & $42 \cdot 43$ \\
\hline Female sex ( $n$ and \%) & 738 & $50 \cdot 34$ \\
\hline \multicolumn{3}{|l|}{ Parent education ( $n$ and \%) } \\
\hline High school or less & 982 & $67 \cdot 17$ \\
\hline More than high school diploma & 480 & $32 \cdot 83$ \\
\hline \multicolumn{3}{|l|}{ Household income ( $n$ and \%) } \\
\hline$<\$ 30 \mathrm{k}$ & 1031 & 71.70 \\
\hline$\geq \$ 30 \mathrm{k}$ & 407 & $28 \cdot 30$ \\
\hline \multicolumn{3}{|l|}{ Youth Hispanic background ( $n$ and \%) } \\
\hline Mexican & 709 & $48 \cdot 66$ \\
\hline Cuban, Dominican or Puerto Rican & 470 & $32 \cdot 26$ \\
\hline Central/South American, mixed, other & 278 & $19 \cdot 08$ \\
\hline \multicolumn{3}{|l|}{ Family dynamics (mean and SD) } \\
\hline Low family function $\ddagger$, score range 1-4 & 1.94 & 0.44 \\
\hline $\begin{array}{l}\text { Family closeness§, score range } 10-30 \\
\text { points }(n 1237)\end{array}$ & $27 \cdot 07$ & $3 \cdot 28$ \\
\hline \multicolumn{3}{|l|}{ Home environment } \\
\hline \multicolumn{3}{|l|}{ Parenting styles ( $n$ and \%) } \\
\hline Authoritative & 610 & 41.72 \\
\hline Neglectful & 511 & 34.95 \\
\hline Indulgent & 130 & 8.89 \\
\hline Authoritarian & 211 & 14.43 \\
\hline $\begin{array}{l}\text { Family support for fruits and vegetables, } \\
\text { score range } 4-20 \text { (mean and SD) }\end{array}$ & $13 \cdot 42$ & 4.35 \\
\hline $\begin{array}{l}\text { Parenting strategies for eating and activity, } \\
\text { score range } 28-127 \text { (mean and SD) }\end{array}$ & 76.93 & $19 \cdot 41$ \\
\hline \multicolumn{3}{|l|}{ Youth eating behaviours } \\
\hline Fruit (servings/d) (median and IQR) & 0.50 & $0.0-1 \cdot 11$ \\
\hline Vegetables (servings/d) (median and IQR) & $1 \cdot 26$ & $0.61-2.12$ \\
\hline $\begin{array}{l}\text { Empty energy content (mean percentage } \\
\text { from energy and SD) }\end{array}$ & $27 \cdot 12$ & 7.57 \\
\hline
\end{tabular}

IQR, interquartile range.

${ }^{*}$ Higher values indicate higher household food security. †Higher values indicate greater acculturative stress on the youth. fHigher values indicate poorer family function.

$\S$ Reported for youth whose mothers or fathers were present. 


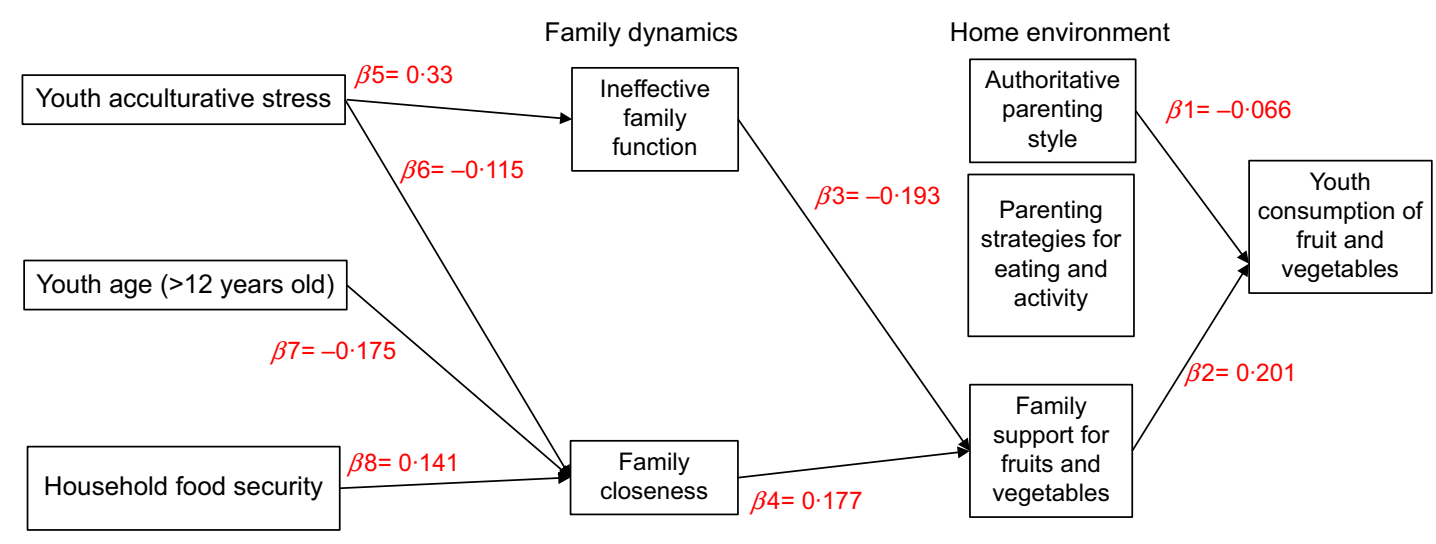

Fig. 2 (colour online) Results from path analysis model on the consumption of fruits and vegetables among Hispanic/Latino youth. Study of Latino Youth. Only significant $(P<0.05)$ pathways are presented. Indirect effects: $\beta 3 \times \beta 2=-0.039(P<0.001)$, $\beta 4 \times \beta 2=0.036 \quad(P<0.001), \quad \beta 5 \times \beta 3 \times \beta 2=-0.013 \quad(P<0.001), \quad \beta 6 \times \beta 4 \times \beta 2=-0.004 \quad(P=0.004), \quad \beta 7 \times \beta 4 \times \beta 2=-0.006$ $(P<0.001), \beta 8 \times \beta 4 \times \beta 2=0.005(P=0.003)$. Youth's gender, parent education and parent Hispanic group were controlled in the model. Model fit statistics: $\chi^{2}=0.454$;f $=2 ; P=0.80 ; \mathrm{CFI}=1 ; \mathrm{TLI}=1.08$; RMSEA $=0.000,90 \% \mathrm{Cl} 0.000,0.033$; WRMR 0.065

(CI) $0.000,0.033$; weighted root mean square residual (WRMR) 0.065). Youth gender, parent education, household income and parent Hispanic subgroups were not significantly associated with family dynamics and therefore are not depicted in the results. Youth FV consumption was directly associated with two scales of the food-related home environment: family support for FV $(\beta 1=0.201, P<0 \cdot 001)$ and an authoritative parenting style $(\beta 2=-0.066$, $P<0.001)$. This can be interpreted as an increase of one unit in the score for family support for FV was associated with a $0 \cdot 2$ increase in servings of $\mathrm{FV}$ per $\mathrm{d}$, whereas an authoritative parenting style (compared with other styles of parenting) was associated with a decrease of 0.066 servings of FV per $\mathrm{d}$, as reported by youth. Youth's perception of ineffective family function was directly negatively associated with youth's perception of family support for FV $(\beta 3=-0 \cdot 193$, $P<0.000)$, while their perception of family closeness was directly associated with higher family support $(\beta 4=0 \cdot 177$, $P<0.001)$. Higher youth acculturative stress was directly associated with lower family function $(\beta 5=0.33$, $P<0.001)$ and lower family closeness $(\beta 6=-0.115$, $P=0.001)$. Being older ( $>12$-year-olds) was associated with perceived lower family closeness $(\beta 7=-0.175, P<0.001)$, while household food security was positively associated with family closeness $(\beta 8=0 \cdot 141, P<0 \cdot 001)$.

Results from the simple mediation effects, family dynamics on youth FV consumption through the foodrelated home environment, suggested that the variables of family dynamics were associated with youth FV intake only via family support and not through the other variables of the home environment. To illustrate, youth's perception of ineffective family function had an inverse association with FV intake via family support $(\beta 3 \times \beta 2=-0.039$, $P<0.001)$. On the other hand, youth's perception of family closeness was positively associated with family support for $\mathrm{FV}$ and greater consumption of youth FV $(\beta 4 \times \beta 2=0.036, P<0.001)$.
Results from the double mediation pathway on youth FV intake through family dynamics and food-related home environment suggested that higher youth acculturative stress was negatively associated with youth FV consumption via a pathway of ineffective family function and lower family support for FV $(\beta 5 \times \beta 3 \times \beta 2=-0.013, P<0 \cdot 001)$. Youth acculturative stress and youth age were also negatively associated with youth FV consumption via youth's perception of family closeness and family support $(\beta 6 \times$ $\beta 4 \times \beta 2=-0.004, P=0.004$ for acculturative stress pathway and $\beta 7 \times \beta 4 \times \beta 2=-0.006, P<0.001$ for youth age pathway). Household food security was indirectly positively associated with FV consumption also via the youth's perception of family closeness and family support for FV $(\beta 8 \times \beta 4 \times \beta 2=0.005, P=0.003)$.

Empty energies model: The model for empty energies also fits the data very well $\left(\chi^{2}=1.852 ; \mathrm{df}=2 ; P=0.3961\right.$; $\mathrm{CFI}=1 \cdot 00 ; \quad \mathrm{TLI}=1 \cdot 00 ; \quad \mathrm{RMSEA}=0.000,95 \%$ CI 0.000 , 0.052, WRMR 0.140) (Fig. 3). Youth gender, parent education, household income and parent Hispanic subgroups were not significantly associated with family dynamics and therefore are not depicted in the results. PEAS $(\beta=-0.077, \quad P=0.018)$ and family support for $\mathrm{FV}$ $(\beta=-0.131, P=0.001)$ were inversely directly associated with higher consumption of empty energies among youth. Family closeness was directly associated with PEAS $(\beta 3=0.160, P<0.001)$ and with family support for $\mathrm{FV}$ $(\beta 4=0.168, P<0 \cdot 001)$. Ineffective family function was inversely directly associated with family support for FV $(\beta 5=-0 \cdot 189, P<0 \cdot 001)$. Youth age and youth acculturative stress were inversely associated with youth's perception of family closeness $(\beta 6=-0 \cdot 175, P<0 \cdot 001$ for youth age and $\beta 8=-0 \cdot 115, P=0 \cdot 001$ for acculturative stress), whereas household food security and youth acculturative stress were directly positively associated with family closeness ( $\beta 7=0 \cdot 141, P<0 \cdot 001$ for household food security and $\beta 9=0.330, P<0.001$ for youth acculturative stress). 
Family dynamics

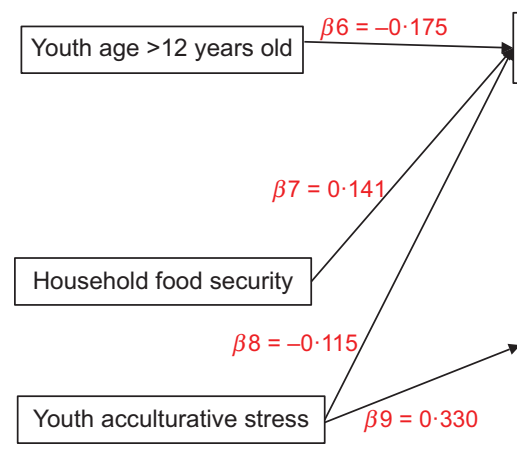

Home environment

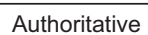

parenting style

Fig. 3 (colour online) Results from path analysis model on consumption of empty energies among Hispanic/Latino youth. Study of Latinos Youth Study. Only significant $(P<0.05)$ pathways are presented. Indirect effects: $\beta 3 \times \beta 1=-0.012(P=0.031)$, $\beta 4 \times \beta 2=-0.022(P=0.004), \quad \beta 5 \times \beta 2=0.025 \quad(P=0.002), \quad \beta 6 \times \beta 3 \times \beta 1=0.002 \quad(P=0.049), \quad \beta 6 \times \beta 4 \times \beta 2=0.004 \quad(P=0.016)$, $\beta 7 \times \beta 4 \times \beta 2=-0.003(P=0.021), \beta 8 \times \beta 4 \times \beta 2=0.003(P=0.028), \beta 9 \times \beta 5 \times \beta 2=0.008(P=0.005)$. Youth gender, parent education, house hold income and parent Hispanic group were controlled in the model. Model fit statistics: $\chi^{2}=1.852 ; \mathrm{df}=2 ; P=0.3961$; $\mathrm{CFI}=1.00 ; \mathrm{TLI}=1.00 ; \mathrm{RMSEA}=0.000,95 \% \mathrm{Cl} 0.000,0.052$; WRMR 0.140

The indirect effects (simple and double mediations) of sociocultural, economic, psychological context and family dynamics on youth empty energies consumption through the food-related home environment are depicted in the subscripts of Fig. 3. Family closeness was negatively associated with empty energies consumption via PEAS $(\beta 3 \times \beta 1=-0.012, P=0.031)$. Weaker family dynamics were also associated with greater consumption of empty energies via lower family support $(\beta 4 \times \beta 2=-0.022, P=0.004$ for family closeness pathway and $\beta 5 \times \beta 2=0.025, P=0.002$ for ineffective family function pathway).

Older youth consumed more empty energies via two pathways, both related to lower family closeness: family closeness and PEAS $(\beta 6 \times \beta 3 \times \beta 1=0.002, P=0.049)$ and family closeness and family support $(\beta 6 \times \beta 4 \times \beta 2=0.004$, $P=0.016$ ). Household food security was inversely associated with youth energy consumption via a pathway of family closeness and family support for $\mathrm{FV}(\beta 7 \times \beta 4 \times \beta 2=-0.003$, $P=0.021)$. Youth acculturative stress was positively associated with youth energy consumption via two pathways: family closeness and family support $(\beta 8 \times \beta 4 \times \beta 2=0.003$, $P=0.028)$ and via ineffective family function and lower family support for $\mathrm{FV}(\beta 9 \times \beta 5 \times \beta 2=0.008, P=0.005)$.

\section{Discussion}

We tested a model that positioned the sociocultural, economic and psychological contexts in which family dynamics may influence youth's eating behaviours via the home environment in a cohort of Hispanic youth. Our analyses partly supported the proposed model: better family dynamics (effective function/closeness) were associated with better home environment (family support for FV and PEAS, but not authoritative parenting style) and youth dietary consumption in the expected directions: higher family function/closeness was associated with higher FV consumption via a pathway of family support for FV and PEAS; weaker family dynamics were associated with increased empty energies consumption via lower family support for FV and PEAS. Age and acculturative stress among youth and lower household food security indirectly influenced the hypothesised pathways in the expected directions.

The findings are congruent with Family Systems Theory, which posits that under conditions of weaker family functioning (e.g. less structure/rules, warmth/communication and problem-solving skills), youth may become more vulnerable to risk behaviours (e.g. poorer diet) ${ }^{(70)}$. The findings from the model are also in agreement with studies in other populations that have examined the relationship between level of family functioning and FV intake among youth, in which low family functioning is associated with inadequate $\mathrm{FV}$ intake and a diet high in red and processed meats, takeaway foods, confectionery and refined foods ${ }^{(41,71-73)}$.

The social-ecological approach posits that family dynamics are nested within socio-economic and cultural contexts. In support of this, the model findings suggest that family dynamics were indirectly associated with youth consumption: perceiving higher acculturative stress was indirectly associated with lower FV consumption and higher consumption of empty energies via a pathway of ineffective family dynamics and lower family support for FV and PEAS. Acculturative stress, defined as psychological, somatic and social difficulties that accompany the adaptation to the new culture ${ }^{(61,62)}$, has been identified as a key source of psychological distress among Hispanic immigrants, associated with depression, anxiety and marital dis$\operatorname{cord}^{(74-76)}$. Acculturative stress can lead to disruptions in family dynamics and relationships, potentially undermining parenting confidence ${ }^{(77)}$ which, in turn, can negatively 
influence behaviours related to youth well-being ${ }^{(78)}$. Low family functioning, potentially arising from these stressors, may then lead to lack of support for healthful eating, as our findings suggest. Although our study hypothesised that acculturative stress influenced weak family dynamics, the association could very well be bidirectional. Ineffective family function may be one aspect of family conflict, and acculturative stress measures also incorporate family acculturation conflicts ${ }^{(79)}$.

Acculturative stress is associated with food insecurity and family support systems: compared with peers in food-secure households, Hispanic youth in food-insecure households experienced greater parent/child acculturative and economic stress and weakened family support systems $^{(46)}$. This is congruent with our findings that higher household food security was positively associated with FV consumption via a pathway of family closeness and family support for FV. Food insecurity may lead to psychological distress because parents feel that they do not have enough to feed their children ${ }^{(80-82)}$. Among immigrants, food insecurity may be exacerbated by the perception of limited access to culturally acceptable foods, in addition to low social support and less time for healthy food planning and preparation compared with prior to immigration $^{(61,62,83,84)}$. However, a study on this same target population of Hispanic youth documented that parental stressors were associated with risk for youth obesity independent of the home food environment and child diet quality $^{(85)}$, suggesting prospective studies to fully understand the temporal nature of these associations and mediators by behavioural risk factors for childhood obesity, including food insecurity and family dynamics.

The pathway between family dynamics and the home environment on eating behaviours seems to be shaped in part by age. Specifically, older age (>12-year-olds) was associated with lower FV consumption and higher empty energy consumption via the hypothesised pathways of family dynamics and home environment. Compared with younger youth, the influence of the home environment among adolescents is weaker and may compete with external influences ${ }^{(86)}$. Adolescents' poor food choices have been interpreted as an act of defiance to parental authority, seeking greater independence and autonomy to make their own decisions, and acceptance by their peer group ${ }^{(87)}$. Peer pressure and need for acceptance and conformity are important predictors of adolescents' purchase and consumption of empty energies ${ }^{(88,89)}$.

Our results suggest that the home environment as defined by food parenting practices but not by parenting styles was significantly associated with higher FV intake and lower energy intake. Our finding of a small but negative direct association between authoritative parenting style and youth FV intake is contrary to previous research and theory $^{(90)}$. A possible explanation may be that Hispanic parenting is a 'non-traditional' mixture of authoritarian and authoritative styles, compared with the dominant culture, as others have described ${ }^{(34,35,91,92)}$. To assist with the interpretation of our results, we ran post hoc analyses using continuous variables of responsiveness and demandingness, but did not find that either subscale was significantly associated with consumption variables. Some researchers question the universal suitability of the parenting style scale to characterise other cultures' parenting styles, since the scale was developed largely for middleclass European Americans ${ }^{(35)}$. Others have suggested adding a bidirectional dimension to the parenting style scale to characterise how youth accept and interact with the parenting styles in order to better understand the dynamics of family and home environments on youth $\operatorname{diet}^{(93)}$.

\section{Strengths and limitations}

The current study should be interpreted in light of its strengths and limitations. First, the study used crosssectional data to test hypothesised mediation paths. We did not test all pathways in the model, and all causal paths were hypothesised to be in one direction even though bidirectional associations are plausible. Although this is the first study to examine such paths between socio-demographic variables, family dynamics and home environment using quantitative data, and based on theory, the hypothesised paths must be further examined using longitudinal data to test if the proposed paths may actually be bidirectional or reversed. Second, there are limitations inherent to selfreported data. Subjective self-ratings of some of the predictor variables may vary in meaning across individuals, since what one person considers to be high, another might consider to be low. However, we did analyse both parent-reported and youth-reported subjective measures for family function, PEAS and noted the medians and distributions in scores were similar between parent and youth reports. Self-reported dietary data are particularly challenging to collect accurately, especially among youth and racial/ethnic minority populations, and the validity of self-reported 24-h recalls has been called into question previously $^{(94)}$. However, interviewer-assisted multiple 24-h recall data have recognised strengths related to increased accuracy and specificity in describing mean values for groups $^{(95)}$. In addition, the current study did not control for other variables that may be important effect modifiers or mediators such as parent or youth depression, or youth school or work hours. In line with that, the analyses could have also reported on direct associations between social/ economic context and parenting strategies or youth FV, but it purposefully does not because the premise of this specific study was to test the hypothesis that the demographic, psychological and economic contexts can indirectly associate with youth FV via family dynamics and home food environment. The study also has notable strengths including a large probability-based sample that 
represents varied heritage and socio-economic facets of the US Hispanic population.

\section{Conclusion}

Our findings suggest that family dynamics indirectly relate to youth's eating behaviours via parenting rules and practices towards a healthy lifestyle (family support for FV and PEAS) and that these paths also depend on youth's age, food security and acculturative stress. The pathways were statistically significant, but in general, predictors had a low magnitude, which suggests that other factors outside of the family environment are directly or indirectly influencing youth's dietary consumption. Whether these associations are evident in longitudinal research is needed to better evaluate strategies to address sociocultural, economic and psychological factors structures that influence family dynamics, the home environment and eating behaviours. The current study is a first step in elucidating complex system relationships to support healthful eating around youth.

\section{Acknowledgements}

Acknowledgements: The authors thank the staff and participants of the HCHS/SOL and SOL Youth for their important contributions. The SOL Youth Study was supported by grant no. R01HL102130 from the National Heart, Lung, and Blood Institute. The youths in SOL Youth are drawn from the study of adults: The HCHS/SOL, which was supported by contracts from the National Heart, Lung, and Blood Institute to the University of North Carolina (N01-HC65233), University of Miami (N01-HC65234), Albert Einstein College of Medicine (N01-HC65235), Northwestern University (N01-HC65236) and San Diego State University (N01-HC65237). The following institutes/ centres/offices contribute to the HCHS/SOL through a transfer of funds to National Heart, Lung, and Blood Institute: National Center on Minority Health and Health Disparities, the National Institute of Deafness and Other Communications Disorders, the National Institute of Dental and Craniofacial Research, the National Institute of Diabetes and Digestive and Kidney Diseases, the National Institute of Neurological Disorders and Stroke and the Office of Dietary Supplements. Additional support was provided by the Life Course Methodology Core of the New York Regional Center for Diabetes Translation Research (DK111022-8786). The study sponsors did not have any role in study design; collection, analysis and interpretation of data; writing the report and the decision to submit the report for publication. The content is solely the responsibility of the authors and does not necessarily represent the official views of the National Heart, Lung, and Blood Institute or the National Institutes of Health. Financial support: U.C.-R. received financial support from the Robert Wood Johnson Foundation (award no. ECNS91160N) for additional training in the methodology used and from the Clinical and Translational Science Institute at Children's National in partnership with the George Washington University voucher award. Conflict of interests: The authors have no conflicts of interest to report. Authorship: U.C.-R. conceived the study question, conceptual framework and study design with significant input from co-authors and mentors (R.M.-R., V.S.C., L.C.G., C.R.I. and D.S.-A.), Y.I.C. and J.W. analysed the data and contributed to interpretation. K.M.P., L.V.H. and all authors contributed significantly to the interpretation of data and manuscript writing. Ethics of human subject participation: The current study was conducted according to the guidelines laid down in the Declaration of Helsinki, and all procedures involving human subjects/patients were approved by the institutional review boards of each of the institutions involved in the study of $\mathrm{HCHS} / \mathrm{SOL}$. Written informed consent and assent were obtained from all parent/caregivers and their youth, respectively.

\section{References}

1. Ogden CL, Carroll MD, Lawman HG et al. (2016) Trends in obesity prevalence among children and adolescents in the United States, 1988-1994 through 2013-2014. JAMA 315, 2292-2299.

2. Lee JM, Okumura MJ, Davis MM et al. (2006) Prevalence and determinants of insulin resistance among U.S. adolescents: a population-based study. Diabetes Care 29, 2427-2432.

3. Hu FB, Manson JE, Stampfer MJ et al. (2001) Diet, lifestyle, and the risk of type 2 diabetes mellitus in women. $N$ Engl J Med 345, 790-797.

4. Stampfer MJ, Hu FB, Manson JE et al. (2000) Primary prevention of coronary heart disease in women through diet and lifestyle. $N$ Engl J Med 1, 16-22.

5. Mozaffarian D, Kamineni A, Carnethon M et al. (2009) Lifestyle risk factors and new-onset diabetes mellitus in older adults: the cardiovascular health study. Arch Intern Med 8, 798-807.

6. Rhee JJ, Mattei J, Hughes MD et al. (2015) Dietary diabetes risk reduction score, race and ethnicity, and risk of type 2 diabetes in women. Diabetes Care 38, 596-603.

7. Templeton SB, Marlette MA \& Panemangalore M (2005) Competitive foods increase the intake of energy and decrease the intake of certain nutrients by adolescents consuming school lunch. J Am Diet Assoc 105, 215-220.

8. Department of Health and Human Services (2015) Dietary Guidelines for Americans 2015-2020. https://health.gov/ dietaryguidelines/2015/guidelines/ (accessed June 2020).

9. Hanson NI, Neumark-Sztainer D, Eisenberg ME et al. (2005) Associations between parental report of the home food environment and adolescent intakes of fruits, vegetables and dairy foods. Public Health Nutr 8, 77-85.

10. Boutelle KN, Fulkerson JA, Neumark-Sztainer D et al. (2007) Fast food for family meals: relationships with parent and 
adolescent food intake, home food availability and weight status. Public Health Nutr 10, 16-23.

11. Campbell KJ, Crawford DA, Salmon J et al. (2007) Associations between the home food environment and obesity-promoting eating behaviors in adolescence. Obesity 15, 719-730.

12. Arcan C, Neumark-Sztainer D, Hannan P et al. (2007) Parental eating behaviours, home food environment and adolescent intakes of fruits, vegetables and dairy foods: longitudinal findings from Project EAT. Public Health Nutr 10, $1257-1265$.

13. Vaughn AE, Ward DS, Fisher JO et al. (2016) Fundamental constructs in food parenting practices: a content map to guide future research. Nutr Rev 74, 98-117.

14. Yee AZ, Lwin MO \& Ho SS (2017) The influence of parental practices on child promotive and preventive food consumption behaviors: a systematic review and meta-analysis. Int J Behav Nutr Phys Act 14, 47.

15. Baumrind D (1966) Effects of authoritative parental control on child behavior. Child Dev 37, 887-907.

16. Brown R \& Ogden J (2004) Children's eating attitudes and behaviour: a study of the modelling and control theories of parental influence. Health Educ Res 19, 261-271.

17. Joyce JL \& Zimmer-Gembeck MJ (2009) Parent feeding restriction and child weight. The mediating role of child disinhibited eating and the moderating role of the parenting context. Appetite 52, 726-734.

18. Larsen JK, Hermans RC, Sleddens EF et al. (2015) How parental dietary behavior and food parenting practices affect children's dietary behavior. Interacting sources of influence? Appetite 89, 246-257.

19. Vereecken CA, Keukelier E \& Maes L (2004) Influence of mother's educational level on food parenting practices and food habits of young children. Appetite 43, 93-103.

20. Vereecken C, Rovner A \& Maes L (2010) Associations of parenting styles, parental feeding practices and child characteristics with young children's fruit and vegetable consumption. Appetite 55, 589-596.

21. Sleddens EF, Gerards SM, Thijs C et al. (2011) General parenting, childhood overweight and obesity-inducing behaviors: a review. Int J Pediatr Obes 6, e12-e27.

22. Gevers DW, Kremers SP, de Vries NK et al. (2015) Patterns of food parenting practices and children's intake of energydense snack foods. Nutrients 7, 4093-4106.

23. Gevers DW, van Assema P, Sleddens EF et al. (2015) Associations between general parenting, restrictive snacking rules, and adolescent's snack intake. The roles of fathers and mothers and interparental congruence. Appetite 87, 184-191.

24. LeCroy MN, Siega-Riz AM, Albrecht SS et al. (2019) Association of food parenting practice patterns with obesogenic dietary intake in Hispanic/Latino youth: results from the Hispanic Community Children's Health Study/Study of Latino Youth (SOL Youth). Appetite 140, 277-287.

25. Arredondo EM, Elder JP, Ayala GX et al. (2006) Is parenting style related to children's healthy eating and physical activity in Latino families? Health Educ Res 21, 862-871.

26. Larios SE, Ayala GX, Arredondo EM et al. (2009) Development and validation of a scale to measure Latino parenting strategies related to children's obesigenic behaviors. The parenting strategies for eating and activity scale (PEAS). Appetite 52, 166-172.

27. Olvera N \& Power TG (2010) Brief report: parenting styles and obesity in Mexican American children: a longitudinal study. J Pediatr Psychol 35, 243-249.

28. Tovar A, Hennessy E, Pirie A et al. (2012) Feeding styles and child weight status among recent immigrant mother-child dyads. Int J Behav Nutr Phys Act 9, 62.

29. Hughes SO, Anderson CB, Power TG et al. Measuring feeding in low-income African-American and Hispanic parents. Appetite 46, 215-223.
30. Hughes SO, Power TG, Orlet Fisher J et al. (2005) Revisiting a neglected construct: parenting styles in a child-feeding context. Appetite 44, 83-92.

31. Hughes SO, Power TG, O'Connor TM et al. (2016) Maternal feeding styles and food parenting practices as predictors of longitudinal changes in weight status in Hispanic preschoolers from low-income families. J Obes 2016, 7201082.

32. Arlinghaus KR, Vollrath K, Hernandez DC et al. (2018) Authoritative parent feeding style is associated with better child dietary quality at dinner among low-income minority families. Am J Clin Nutr 108, 730-736.

33. Roche K, Ensminger M \& Cherlin A (2007) Variations in parenting and adolescent outcomes among African American and Latino families living in low-income, urban areas. J Fam Issues 28, 882-909.

34. Domenech Rodriguez MM, Donovick MR \& Crowley SL (2009) Parenting styles in a cultural context: observations of "protective parenting" in first-generation Latinos. Fam Process 48, 195-210.

35. Driscoll AK, Russell ST \& Crockett LJ (2008) Parenting styles and youth well-being across immigrant generations. J Fam Issues 20, 185-209.

36. Kerr ME (2000) "One Family's Story: A Primer on Bowen Theory." The Bowen Center for the Study of the Family. http://www.thebowencenter.org (accessed June 2020).

37. Byles J, Byrne C, Boyle MH et al. (1988) Ontario Child Health Study: reliability and validity of the general functioning subscale of the McMaster Family Assessment Device. Fam Process 27, 97-104.

38. Epstein NB, Baldwin LM \& Bishop DS (1983) The McMaster Family Assessment Device. J Marital Fam Ther 3, 171-180.

39. Kapinus C \& Corman B (2004) Closeness with parents and perceived consequences of pregnancy among male and female adolescents. Sociol $Q \mathbf{4 5}, 691-717$.

40. Renzaho AM, Dau A, Cyril S et al. (2014) The influence of family functioning on the consumption of unhealthy foods and beverages among 1- to 12-y-old children in Victoria, Australia. Nutrition 30, 1028-1033.

41. Renzaho AM, Kumanyika S \& Tucker KL (2011) Family functioning, parental psychological distress, child behavioural problems, socio-economic disadvantage and fruit and vegetable consumption among 4-12 year-old Victorians, Australia. Health Promot Int 26, 263-275.

42. Wen LM, Simpson JM, Baur LA et al. (2011) Family functioning and obesity risk behaviors: implications for early obesity intervention. Obesity 19, 1252-1258.

43. Arandia G, Sotres-Alvarez D, Siega-Riz AM et al. (2018) Associations between acculturation, ethnic identity, and diet quality among U.S. Hispanic/Latino Youth: findings from the HCHS/SOL Youth Study. Appetite 129, 25-36.

44. Varela RE, Vernberg EM, Sanchez-Sosa JJ et al. (2004) Parenting style of Mexican, Mexican American, and Caucasian-non-Hispanic families: social context and cultural influences. J Fam Psychol 18, 651-657.

45. Varela RE, Sanchez-Sosa JJ, Biggs BK et al. (2009) Parenting strategies and socio-cultural influences in childhood anxiety: Mexican, Latin American descent, and European American families. J Anxiety Disord 23, 609-616.

46. Potochnick S, Perreira KM, Bravin JI et al. (2019) Food insecurity among Hispanic/Latino youth: who is at risk and what are the health correlates? J Adolesc Health 64, 631-639.

47. Mozaffarian D, Angell SY, Lang T et al. (2018) Role of government policy in nutrition-barriers to and opportunities for healthier eating. BMJ 361, k2426.

48. Bronfrebrenner U (1981) The Ecology of Human Development: Experiments by Nature and Design. Boston, MA: Harvard University Press.

49. Isasi CR, Carnethon MR, Ayala GX et al. (2014) The Hispanic Community Children's Health Study/Study of Latino Youth 
(SOL Youth): design, objectives, and procedures. Ann Epidemiol 24, 29-35.

50. Sorlie PD, Aviles-Santa LM, Wassertheil-Smoller S et al. (2010) Design and implementation of the Hispanic Community Health Study/Study of Latinos. Ann Epidemiol 20, 629-641.

51. Ayala GX, Carnethon M, Arredondo E et al. Theoretical foundations of the Study of Latino (SOL) Youth: implications for obesity and cardiometabolic risk. Ann Epidemiol 24, 36-43.

52. Lavange LM, Kalsbeek WD, Sorlie PD et al. (2010) Sample design and cohort selection in the Hispanic Community Health Study/Study of Latinos. Ann Epidemiol 20, 642-649.

53. Feskanich D, Buzzard I, Welch B et al. (1988) Comparison of a computerized and a manual method of food coding for nutrient intake studies. J Am Diet Assoc 88, 1263-1267.

54. University of Minnesota (2018) NCC Food and Nutrient Database Version 2018. Nutrition Data System for Research, Appendix 20 Frequently Asked Questions. University of Minnesota, p. A20.7-8.

55. Miller PE, Mitchell DC, Harala PL et al. (2011) Development and evaluation of a method for calculating the Healthy Eating Index-2005 using the Nutrition Data System for Research. Public Health Nutr 14, 306-313.

56. Guenther PM, Reedy J \& Krebs-Smith SM (2008) Development of the healthy eating index-2005. J Am Diet Assoc 108, 1896-1901.

57. Perreira KM, Marchante AN, Schwartz SJ et al. (2019) Stress and resilience: key correlates of mental health and substance use in the Hispanic community health study of Latino youth. J Immigr Minor Health 21, 4-13.

58. Burgess-Champoux TL, Larson N, Neumark-Sztainer D et al. (2009) Are family meal patterns associated with overall diet quality during the transition from early to middle adolescence? J Nutr Educ Behav 41, 79-86.

59. Norman GJ, Carlson JA, Sallis JF et al. (2010) Reliability and validity of brief psychosocial measures related to dietary behaviors. Int J Behav Nutr Phys Act 7, 56.

60. Jackson C, Henriksen L \& Foshee VA (1998) The authoritative parenting index: predicting health risk behaviors among children and adolescents. Health Educ Behav 25, 319-337.

61. Tovar A, Must A, Metayer N et al. (2013) Immigrating to the US: what Brazilian, Latin American and Haitian women have to say about changes to their lifestyle that may be associated with obesity. J Immigr Minor Health 15, 357-364.

62. Sussner KM, Lindsay AC, Greaney ML et al. (2008) The influence of immigrant status and acculturation on the development of overweight in Latino families: a qualitative study. J Immigr Minor Health 10, 497-505.

63. Gil AG \& Vega WA (1996) Two different worlds: acculturation stress and adaptation among Cuban and Nicaraguan families. J Soc Pers Relat 13, 435.

64. Gil AG, Vega WA \& Dimas JM (1994) Acculturative stress and personal adjustment among Hispanic adolescent boys. J Commun Psychol 22, 43-54

65. Muthen LK \& Muthen BO (1998-2017) MPlus User's Guide, 7 th ed. Los Angeles, CA: Muthen \& Muthen.

66. Bollen KA \& Stine RA (1992) Bootstrapping goodness-of-fit measures in structural equation models. Sociol Methods Res 21, 205-229.

67. Muthen LK \& Muthen BO (2016) MPlus User's Guide, 8th ed. Los Angeles, CA: Muthen \& Muthen.

68. Arbuckle JL (1996) Full information estimation in the presence of incomplete data. In Advanced Structural Equation Modeling: Issues and Techniques, pp. 243-277 [Marcoulis GaSRE, editor] New York: Taylor \& Francis Group, Psychology Press.

69. Little RJ \& Rubin DB (2002) Bayes and multiple imputation. In Statistical Analysis with Missing Data, pp. 200-220. New
Jersey: Wiley Interscience: John While \& Sons Inc Publication.

70. Berge JM, Wall M, Larson $\mathrm{N}$ et al. (2013) Family functioning: associations with weight status, eating behaviors, and physical activity in adolescents. $J$ Adolesc Health 52, 351-357.

71. Ambrosini GL, Oddy WH, Robinson M et al. (2016) Adolescent dietary patterns are associated with lifestyle and family psycho-social factors - CORRIGENDUM. Public Health Nutr 19, 765.

72. Ambrosini GL, Oddy WH, Robinson M et al. (2009) Adolescent dietary patterns are associated with lifestyle and family psycho-social factors. Public Health Nutr 12, $1807-1815$.

73. Oddy WH, Robinson M, Ambrosini GL et al. (2009) The association between dietary patterns and mental health in early adolescence. J Prev Med 49, 39-44.

74. Dillon FR, De La Rosa M \& Ibanez GE (2013) Acculturative stress and diminishing family cohesion among recent Latino immigrants. J Immigr Minor Health 15, 484-491.

75. Revollo HW, Qureshi A, Collazos F et al. (2011) Acculturative stress as a risk factor of depression and anxiety in the Latin American immigrant population. Int Rev Psychiatry 23, 84-92.

76. Leidy MS, Parke RD, Cladis M et al. (2009) Positive marital quality, acculturative stress, and child outcomes among Mexican Americans. J Marriage Fam 71, 833-847.

77. Kiang L, Glatz T \& Buchanan CM (2017) Acculturation conflict, cultural parenting self-efficacy, and perceived parenting competence in Asian American and Latino/a families. Fam Process 56, 943-961.

78. Lorenzo-Blanco EI, Meca A, Unger JB et al. (2016) Latino parent acculturation stress: longitudinal effects on family functioning and youth emotional and behavioral health. J Fam Psychol 30, 966-976.

79. Castillo LG, Cano MA, Chen SW et al. (2008) Family conflict and intragroup marginalization as predictors of acculturative stress in Latino college students. Int J Stress Manag 15, 43-52.

80. Martin MS, Maddocks E, Chen Y et al. (2016) Food insecurity and mental illness: disproportionate impacts in the context of perceived stress and social isolation. Public Health 132, 86-91.

81. Knowles M, Rabinowich J, Ettinger de Cuba S et al. (2016) "Do you wanna breathe or eat?": parent perspectives on child health consequences of food insecurity, trade-offs, and toxic stress. Matern Child Health J 20, 25-32.

82. Laraia B, Vinikoor-Imler LC \& Siega-Riz AM (2015) Food insecurity during pregnancy leads to stress, disordered eating, and greater postpartum weight among overweight women. Obesity 23, 1303-1311.

83. Delavari M, Farrelly A, Renzaho A et al. (2013) Experiences of migration and the determinants of obesity among recent Iranian immigrants in Victoria, Australia. Ethn Health 18, 66-82.

84. Hwang JY, Lee SE, Kim SH et al. (2010) Psychological distress is associated with inadequate dietary intake in Vietnamese marriage immigrant women in Korea. $\mathrm{J} \mathrm{Am}$ Diet Assoc 110, 779-785.

85. Isasi CR, Hua S, Jung M et al. (2017) The association of paren$\mathrm{tal} /$ caregiver chronic stress with youth obesity: findings from the study of Latino youth and the Hispanic Community Health Study/Study of Latinos Sociocultural Ancillary Study. J Child Obes 13, 251-258.

86. Reicks M, Banna J, Cluskey M et al. (2015) Influence of parenting practices on eating behaviors of early adolescents during independent eating occasions: implications for obesity prevention. Nutrients $\mathbf{7}, 8783-8801$.

87. Story M, Neumark-Sztainer D \& French S (2002) Individual and environmental influences on adolescent eating behaviors. J Am Diet Assoc 102, S40-S51. 
88. Bassett R, Chapman GE \& Beagan BL. (2008) Autonomy and control: the co-construction of adolescent food choice. Appetite 50, 325-332.

89. Salvy SJ, de la Haye K, Bowker JC et al. (2012) Influence of peers and friends on children's and adolescents' eating and activity behaviors. Physiol Behav 106, 369-378.

90. Collins C, Duncanson K \& Burrows T (2014) A systematic review investigating associations between parenting style and child feeding behaviours. J Hum Nutr Diet 27, 557-568.

91. Falicov CJ (2006) Family organization: the safety net of close and extended kin. In Counseling and Family Therapy with
Latino Populations, pp. 41-62 [RL Smith and RE Montilla, editors]. New York: Taylor \& Francis Group.

92. Ayon C, Williams LR, Marsiglia FF et al. (2015) A latent profile analysis of Latino parenting: the infusion of cultural values on family conflict. Fam Soc 96, 203-210.

93. Lunn TE, Nowson CA, Worsley A et al. (2014) Does personality affect dietary intake? Nutrition 30, 403-409.

94. Trevino RP, Ravelo AV, Senne-Duff B et al. (2016) Poor validity of dietary recall in low-income Hispanic children using digital food imaging analysis as the reference. J Food Nutr Diet 1, 107.

95. Willett W (2012) Nutritional Epidemiology, 3rd ed., 552 p. New York: Oxford University Press. 